\title{
Affordable Dial-up PPP Internet Connection for School Classroom Local Area Networks
}

\author{
Igor Ozimek \\ “Jožef Stefan” Institute, Ljubljana, Slovenia
}

\begin{abstract}
A software package is presented that provides a simple and low cost solution for schools to connect their classroom Local Area Networks (LAN) to Internet and to use Internet services. The main component of the package is NOS4S (NOS for Schools), a DOS program based on JNOS with some substantial extensions necessary for the intended use. Besides acting as an on-demand TCP/IP router over dial-up Public Switched Telephone Network (PSTN) or ISDN lines, the program also serves as a local e-mail (on-demand SMTP / POP3), FTP and WWW server.
\end{abstract}

Although relatively low cost commercial routers for connecting an LAN to Internet can be found on the market, it is generally difficult for schools to afford them due to their limited financial resources. In contrast, the NOS4S solution is essentially free. On the one hand, NOS4S is publicly available and free. On the other hand, because the classroom computers must be periodically replaced with newer ones to be able to run current versions of MS Windows, some older and abandoned computer suitable to run NOS4S can usually be found in a school.

Keywords: classroom LAN, dial-up Internet access, $\mathrm{TCP} / \mathrm{IP}$ router, $\mathrm{SMTP}$ server.

\section{Introduction}

Internet is an important communication medium and source of information, more and more part of everyday life. For it to be used efficiently, it is important that pupils in secondary and also primary schools are properly educated in its use. A prerequisite for that is connection of schools' computer classrooms to the global Internet. The classical solution using a leased line together with commercial routers is expensive and rarely affordable for schools. For dial-up access, there are reasonably priced dial-up routers (a router integrated with an ISDN adapter or analog modem) on the market, but a router is only one part of the story. Additionally, a number of Internet services are desirable - if not necessary - in a school local network, most of all an e-mail server.

There is a strong interest in primary schools to connect computer classrooms to Internet and there are organised activities towards this goal. However, the number of schools is large and funds are limited. The aim of the project "Affordable dial-up PPP/SLIP LAN-to-Internet connection" [1], which is described in this paper, was to create a non-commercial, free solution for connecting primary and secondary school computer classrooms to Internet. This project was a subproject of a joint INCO-Copernicus project, "Cooperative Research in Information Infrastructure", [2]. It comprised two parts. The first dealt with a software solution for routing of Internet traffic between a local area network and a dial-up Internet connection line, as well as with some other basic local Internet services. In the second part, a bundle of publicly available Internet applications for MS Windows was selected and prepared for installation and distribution on a CD-ROM.

In this article, the first part, i.e. the solution for Internet traffic routing and basic Internet services is presented. Named NOS4S, it was created starting from an existing publicly available application, JNOS, which by itself did not match all the requirements.

Sections 2 to 4 give technical background related to routing and e-mail access over dial-up lines. Section 2 presents dial-up access as a cost-effective solution to connect a LAN to the 
Internet, section 3 explains different approaches for routing Internet traffic between machines on a LAN and the global Internet, and section 4 deals with specific issues of accessing e-mail over a non-permanent dial-up Internet connection. Next, section 5 discusses different software solutions for LAN-to-Internet routing and local e-mail service, with emphasis on the KA9Q family of DOS-based programs. Finally, section 6 presents NOS4S, the newly created solution specifically optimised for use in schools.

\section{Using a Dial-up Line to Connect to Internet}

For low-cost and affordable Internet access, dial-up connections are often used, avoiding dedicated and costly leased lines. Only a standard analog (PSTN) or an ISDN phone line is needed in this case, together with a modem or an ISDN adapter.

An analog connection provides transmission speeds of up to $33 \mathrm{~kb} / \mathrm{s}$ from a user to an ISP (Internet Service Provider) (upload) and up to $56 \mathrm{~kb} / \mathrm{s}$ in the opposite direction (download), using a V.90 modem. The actual speed attainable depends on the quality of the phone line. An ISDN connection provides the speed of 64 $\mathrm{kb} / \mathrm{s}$ in both directions, which can be doubled by using both ISDN data channels if the ISP supports this option. Further advantages of ISDN are much faster establishment of connections and resistance to bad line conditions.

For dial-up connections, a special data link layer protocol is used, PPP (RFC 1661 - The Pointto-Point Protocol, for all RFC standards refer to [3]), which can carry various standard network protocols, including TCP/IP (Internet), [4].

Dial-up connections are most often used to connect home computers to the Internet (Fig. 1).

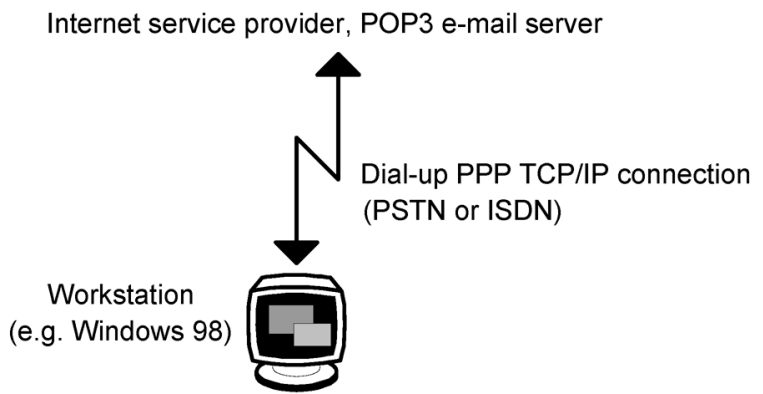

Fig. 1. Individual dial-up Internet connection.
Besides connectivity, an ISP usually also provides an account on its POP3 e-mail server for each user.

Dial-up access can also be used to connect a LAN to the Internet. In this case an additional device, a router, is needed to route traffic between the local network (usually Ethernet) and external Internet. Regarding the e-mail service, where a large number of users is involved which, in addition, change frequently (both certainly hold true for school networks), managing POP3 e-mail accounts for all these users at the ISP site is not very desirable. The problem is avoided by using an e-mail server in the local network and managing the users locally. Fig. 2 shows an example of a local network with two separate devices: a dial-up router and an additional local e-mail server.

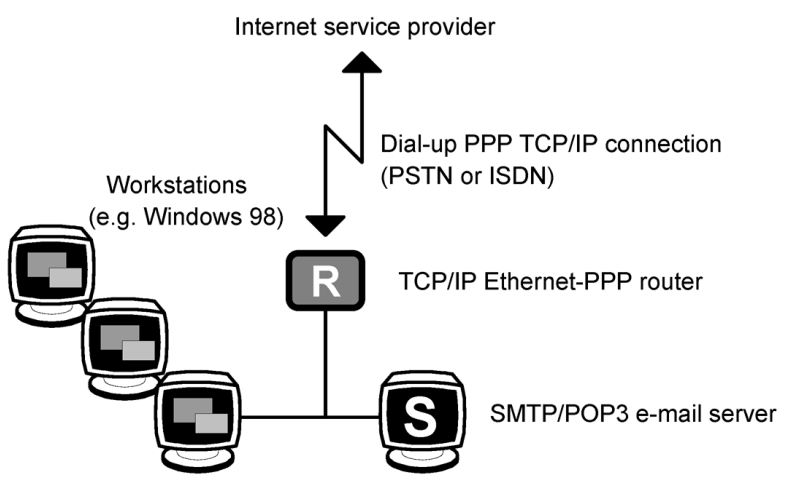

Fig. 2. LAN-to-Internet dial-up connection using a separate router.

A separate router can be avoided. A computer providing the e-mail service (and possibly some other services) in the local network can also, with suitable application software, take over the task of routing (Fig. 3 ).

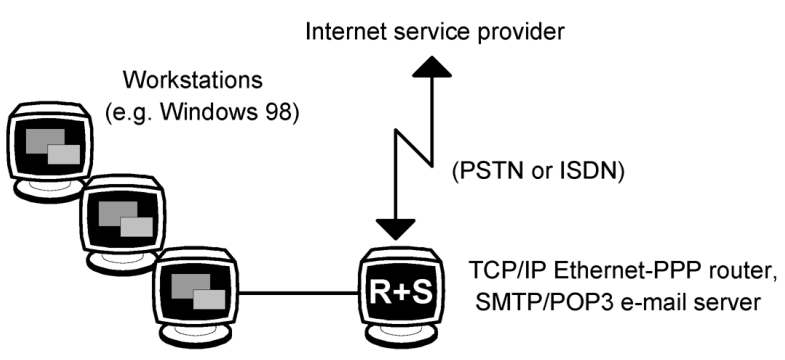

Fig. 3. LAN-to-Internet dial-up connection with software router on a general-purpose computer. 
In the next two chapters we shall take a closer look at the two important functions, routing and e-mail service, and related problems.

\section{IP Routing - Different Approaches}

Different approaches are used for routing Internet (IP) traffic between the global Internet and machines connected to a LAN. Each has its own advantages and drawbacks.

\subsection{Standard IP Routing}

Standard routing requires each machine on the local network to have its own global IP network address. With dial-up access, the usual single-user account, which normally uses a dynamically assigned (non-fixed) IP address, is not adequate. Instead, a special dial-up account must be created for each LAN with a fixed set of IP addresses (a subnet) statically assigned to it.

However, since this solution does not introduce any network "tricks", all current and future network applications work without problem.

\subsection{Proxy Server}

This is a very popular solution, in which a socalled proxy server, as the only machine directly connected to the Internet, acts on behalf of the local LAN-connected hosts at the application level. For example, a WWW browser can be configured to send all its requests to a WWW (HTTP) proxy server. The proxy server resends the requests as its own to the final destinations (WWW servers) in the Internet and delivers all the received replies to the original sender. Although the local LAN hosts use the TCP/IP protocol and have distinct Internet addresses, the only IP address known to the outside world (the global Internet) is that of the proxy server; the whole LAN looks like a single machine and only one global Internet address needs to be registered. The hosts on the LAN could use an arbitrary group of IP addresses, but to minimise the possibility of conflicts due to misconfigured routers, special address spaces have been allocated for unregistered private use by RFC 1918 - Address Allocation for Private Internets, as shown in Table 1.

There are two main advantages in using a proxy server to connect a LAN to the Internet. The first is that a standard single-user dial-up account as normally provided by an ISP can be used. The second is that a proxy server by its nature prevents direct access from Internet to internal machines, thus providing a kind of firewall. At the same time, the proxy server is the natural place for caching service, which enables quicker access to commonly used Internet documents. So, a proxy server usually provides the following additional functions:

- Firewall, access restrictions ([5], RFC 2979 - Behavior of and Requirements for Internet Firewalls). It can be used to selectively restrict the access of internal users to the external Internet.

- HTTP cache. Since a proxy router already relays the WWW traffic (with deep understanding of the protocol) it can make some extra effort and cache the information retrieved so that repeated requests from the same or other browsers are served locally. This can greatly reduce the external WWW traffic, which represents a major bottleneck.

\begin{tabular}{||c|c|c||}
\hline Address range & Subnet class & $\begin{array}{c}\text { No. of } \\
\text { subnets }\end{array}$ \\
\hline \hline $\begin{array}{c}10.0 .0 .0-10.255 .255 .255 \\
(10 / 8 \text {-bit prefix })\end{array}$ & $\begin{array}{c}\mathrm{A} \\
\text { (24-bit node address) }\end{array}$ & 16 \\
\hline $\begin{array}{c}172.16 .0 .0-172.31 .255 .255 \\
(172.16 / 12 \text {-bit prefix })\end{array}$ & $\begin{array}{c}\mathrm{B} \\
(16-\text { bit node address })\end{array}$ & 256 \\
\hline $\begin{array}{c}192.168 .0 .0-192.168 .255 .255 \\
(192.168 / 16 \text {-bit prefix })\end{array}$ & $\begin{array}{c}\mathrm{C} \\
\text { (8-bit node address) }\end{array}$ & \\
\hline
\end{tabular}

Table 1. Address allocation for private Internets (RFC 1918). 
The cache of course requires a considerable amount of disk storage.

However, proxy servers also have certain drawbacks. One is that they work at the application level and must therefore support different applications and their application-level protocols; the applications that are not supported will not work. Often, this application-protocol support is not enough and client software on local hosts must also support working through a proxy server, which is the same as working through a firewall.

The second potential drawback is that LANconnected hosts cannot be used as globally accessible Internet servers. (There is a solution to this: by using mapped links, an internal machine can be defined as a global Internet server for a particular application/protocol.)

Proxy servers support different ways of connecting local applications (clients) to the outside world:

- Gateways (Telnet, FTP). A user first connects to the proxy server and there he/she issues a command to connect to the desired remote server. Graphical FTP clients must explicitly support this mode of operation, which is actually the same as working through a firewall. To make the things a little more complicated, there is not only one but a number of different initial connection procedures used by different proxy servers / firewalls.

(It must be noted that this mode has nothing to do with another option, PASS - Passive. This is required for standard routers and firewalls that, for security reasons, prohibit active connections from a remote FTP server to a local FTP client, which is the normal way of establishing an FTP data connection. The PASS option reverses this direction.)

- Mapped Links (E-mail, News). A mapped link transparently connects traffic of a particular application-level protocol between a client and a remote server. The translation (mapping) of the IP addresses is stored in a table on the proxy server. The client connects to the proxy server and has no option to choose the remote IP address (which is set in the table). Hence, this solution is limited to applications with fixed remote servers like e-mail and news.
- WWW Proxy (HTTP, FTP, GOPHER,... ). As the name implies, it is used specifically for WWW browsers, which must explicitly support this mode of operation. WWW proxy relays all the requests from the browsers and the retrieved information from the remote servers.

- SOCKS ([6], RFC 1928 - SOCKS Protocol Version 5). This is a special service that can be used to establish any kind of connection in a unified way, but a client must explicitly support this type of operation.

\subsection{NAT}

Another approach, which is becoming more and more popular, is NAT (Network Address Translation, [7], [8], RFC 1631 - The IP Network Address Translator (NAT)). Similarly to the proxy server approach, only one Internet address (the NAT router's one) is globally known and accessible and all the external traffic is done in the name of the router and with its Internet address. However, here the mapping is done at the basic IP protocol level and not at the higher application-protocol level. A NAT router looks like a standard IP router/gateway to the local hosts, who automatically send all the outbound traffic to it. The NAT replaces the source IP addresses and port numbers within the packets by its own address and port numbers, and stores this information in a mapping table. Based on this mapping it delivers the received packets to correct local destinations, changing the packets' destination addresses and port numbers appropriately.

Like a proxy server, a NAT router can connect a LAN to the Internet over a standard singleuser dial-up account. The main advantage of NAT is that it is application-independent, since it works at the lower IP level. Hence, there is no need for a specific application-protocol support. (Strictly speaking, this is not completely true, and it can only be said that the NAT approach is more transparent and less applicationdependent than proxy servers. For example, an FTP client initiates a control connection to a FTP server but normally expects the FTP server to initiate the data connections as needed hence, a FTP client acts as a server for data transfer. For this process to be transparent to 
the local client, additional processing at the application protocol level must be performed by a NAT router.)

On the other hand, due to its greater transparency, a NAT router by itself does not provide certain functions inherent in proxy servers, e.g. traffic filtering, access control, caching, etc.

\section{E-mail over a Dial-up Connection}

Two protocols are most commonly used for Internet (TCP/IP) e-mail transfer: SMTP (RFC 821 - Simple Mail Transfer Protocol) and POP3 (RFC 1939 - Post Office Protocol - Version 3).

There is another more advanced and complex protocol, IMAP4 (RFC 1730 - Internet Message Access Protocol - Version 4, see also RFC 2060 - Internet Message Access Protocol - Version 4rev1, RFC 2061 - IMAP4 Compatibility with IMAP2bis), which can be used instead of POP3. Although it is gaining in popularity and most new e-mail clients support it, we shall limit our discussion here, without loss of generality, to POP3 due to its wide-spread use and general adoption.

Neither SMTP nor POP3 were originally designed for the task we want to accomplish, i.e. transferring e-mail of a large number of users on demand. SMTP protocol is used in Internet to transfer e-mail between e-mail servers. The only exception is the final step. Unless a user reads messages directly on an SMTP server, POP 3 or IMAP protocol is normally used to retrieve messages from his/her e-mail box (Fig. 4).

The main reason that renders the original SMTP protocol unsuitable for the final delivery to a personal computer lies in the fact that it was designed for permanently connected and available hosts and not for on-demand e-mail transfer. This is usually not the case with personal computers, which are often switched off when not in use, and many of them use dial-up access rather than permanent Internet connections. When a SMTP host is not available, the sending SMTP server usually keeps trying periodically for a few days and then, if not successful, gives up and sends return information back to the sender.

The POP3 protocol, on the other hand, was designed for on-demand e-mail retrieval but it is suitable mainly for single-user accounts.

In the circumstances where no proper protocol existed for on-demand group e-mail transfer, corresponding solutions were developed for both SMTP and POP3.

The two POP3 solutions are often called MultiPOP and SmartPOP. MultiPOP periodically retrieves the users' e-mail from their individual accounts on the (external) server(s) and makes it available from the local POP3 server. The drawback of this approach is that multiple individual user accounts are required on the Internet provider side. SmartPOP can retrieve all the mail from a single (external) POP3 group account (to which e-mail for a whole group of users is delivered) and delivers it "smartly" (hence the name) according to the headings (e.g. the e-mail address in the "To:" field). This task can be somewhat tricky, e.g. in the case of mailing lists, etc. The advantage is that the Internet provider needs only one user account (actually a group account) to which email messages for a whole group of users are routed.

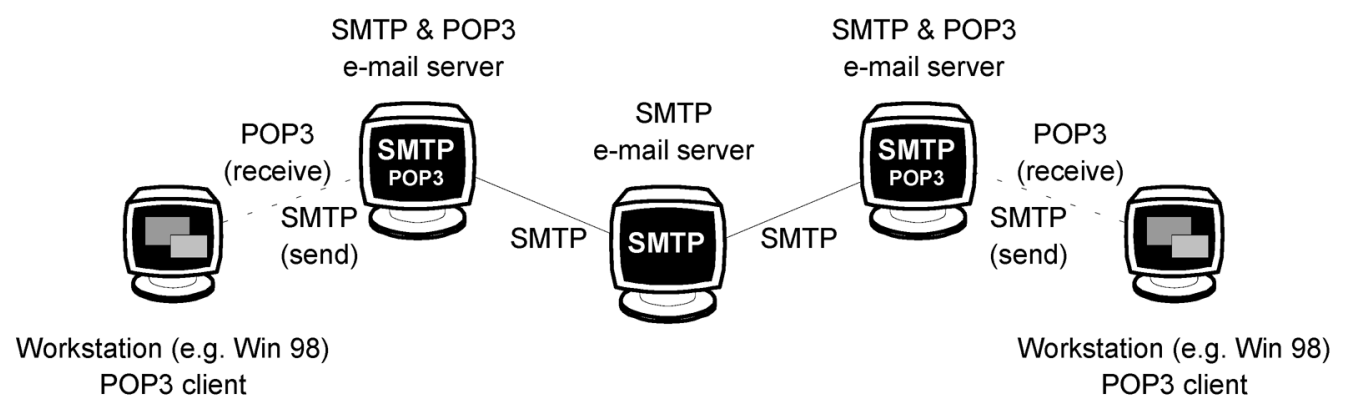

Fig. 4. E-mail transfer by SMTP and POP3 protocols. 
The SMTP on-demand transfer relies on the fact that SMTP servers can be made to begin transmission by different stimuli, e.g. by sending a SMTP command, pinging a port or sending a (dummy) e-mail message. A better solution is to use a special enhanced command ETRN. This command has its roots in an optional command TURN that had been defined in the original RFC 821 SMTP specification. The TURN command suffered from a large security loophole and was generally not implemented. Later, in RFC 1869 - SMTP Service Extensions, a mechanism was added to the SMTP protocol that provided for the capability to extend the command set understood by an SMTP server (called then an ESMTP server). In RFC 1985 - SMTP Service Extension for Remote Message Queue Starting, a new version of the command TURN, ETRN, was defined. Many newer ESMTP servers, like the well-known publicly available sendmail, support this command.

The ETRN command causes an ESMTP server to send immediately all the waiting e-mail message for a specified e-mail subdomain.

\section{Software Solutions for Routing and e-mail Service}

This section deals with choosing or creating a proper software solution for routing and email service. This includes selection of a suitable hardware platform, operating system and application software. With a price-sensitive user in mind (schools), it must be as inexpensive as possible. This rules out any other type of computer but IBM-PC compatibles. Table 2 gives a comparison of different operating systems with expected software prices, hardware requirements and administration complexity. The drawback of UNIX is its administration complexity and the fact that administrators in schools are usually not familiar with this system. The same is true for Netware. Additionally, a Netware solution can be expensive (although schools might be able to obtain the software for an affordable price) and it is losing its market share against MS Windows operating system. MS Windows, on the other hand, requires powerful hardware, and Windows 95/98/ME would not be the best solution due to its limited stability. Finally, DOS, although a very primitive operating system, is simple and inexpensive. It can run on old outdated computers that are not suitable for use with current versions of MS Windows, and that are present in considerable numbers in schools. For these reasons, DOS was chosen as the most appropriate environment for our case.

One might consider DOS an outdated and unsuitable system. However, a number of DOS programs for routing and other functions are still used and are even being further developed. Table 3 gives an overview of publicly available inexpensive or free routing programs for DOS, [9].

Besides the basic TCP/IP routing, at least the following functions are needed:

- automatic on-demand connection establishment and closing,

- e-mail service (SMTP and POP3 servers),

- on-demand e-mail transfer from an ISP's SMTP server.

As seen from Table 3, none of the programs fulfil all the requirements. JNOS matches them most closely (it is the only one which supports both required functions, routing and e-mail service), it is free for non-commercial use, and its source code is publicly available so that it can be tailored to specific needs. It was chosen as the starting point for creating a modified version optimised for our use.

\begin{tabular}{||c|c|c|c||}
\cline { 2 - 4 } \multicolumn{1}{c|}{} & $\begin{array}{c}\text { Application } \\
\text { software price }\end{array}$ & $\begin{array}{c}\text { Hardware } \\
\text { requirements }\end{array}$ & $\begin{array}{c}\text { Administration } \\
\text { complexity }\end{array}$ \\
\hline DOS & free-low & low & low-medium \\
\hline Win 95/98/NT & low-high & high & low-medium \\
\hline UNIX (Linux, FreeBSD) & free-low & medium & high \\
\hline Netware & medium-high & medium & high \\
\hline \hline
\end{tabular}

Table 2. Different IBM-PC-based systems for routing and e-mail service. 


\begin{tabular}{||l||c|c|c|c||}
\cline { 2 - 5 } \multicolumn{1}{c|}{} & PCROUTE & INAR & JNOS & IPROUTE \\
\hline \hline Built-in PPP protocol & - & - & yes & yes \\
\hline SMTP/POP3 & - & - & not on-demand & - \\
\hline Call on demand & - & - & - & yes \\
\hline Source code available & yes & yes & yes & - \\
\hline Price & free & free & free & $\$ 50$ \\
\hline
\end{tabular}

Table 3. TCP/IP routing software applications for DOS environment.

JNOS is only one of a group of programs collectively known under acronym KA9Q or NOS (Network Operating System). The program was originally developed for radio-ham usage and KA9Q is actually the radio-ham call sign of its primary author Phil Karn. KA9Q is much more than a TCP/IP router. It is a complete system with a multitasking kernel and includes a number of servers (FTP, SMTP, POP3, DNS, WWW, Gopher) as well as clients (Telnet, FTP). Many different versions have been developed, supporting somewhat different services and functions. Fig. 5 shows the "family tree" of those versions that are most often found in general (non-radio-ham) use:

- DIS, developed by British ISP (Internet service provider) Demon for its customers,
- CWRU/BIOC, developed and used in the academic environment (universities),

- JNOS, the main radio-ham version, still being further developed, very stable, with a lot of Internet services.

\section{NOS4S - NOS for Schools}

Our version of the program, modified for use in schools, is called NOS4S (NOS for Schools). It is based on the source code of JNOS $1.10 \mathrm{~m}$ with a few parts taken from a newer release $1.11 \mathrm{~b}$. JNOS is written mostly in $\mathrm{C}$ and partly in assembler language and comprises about 400 files of $4.5 \mathrm{MB}$ in length.

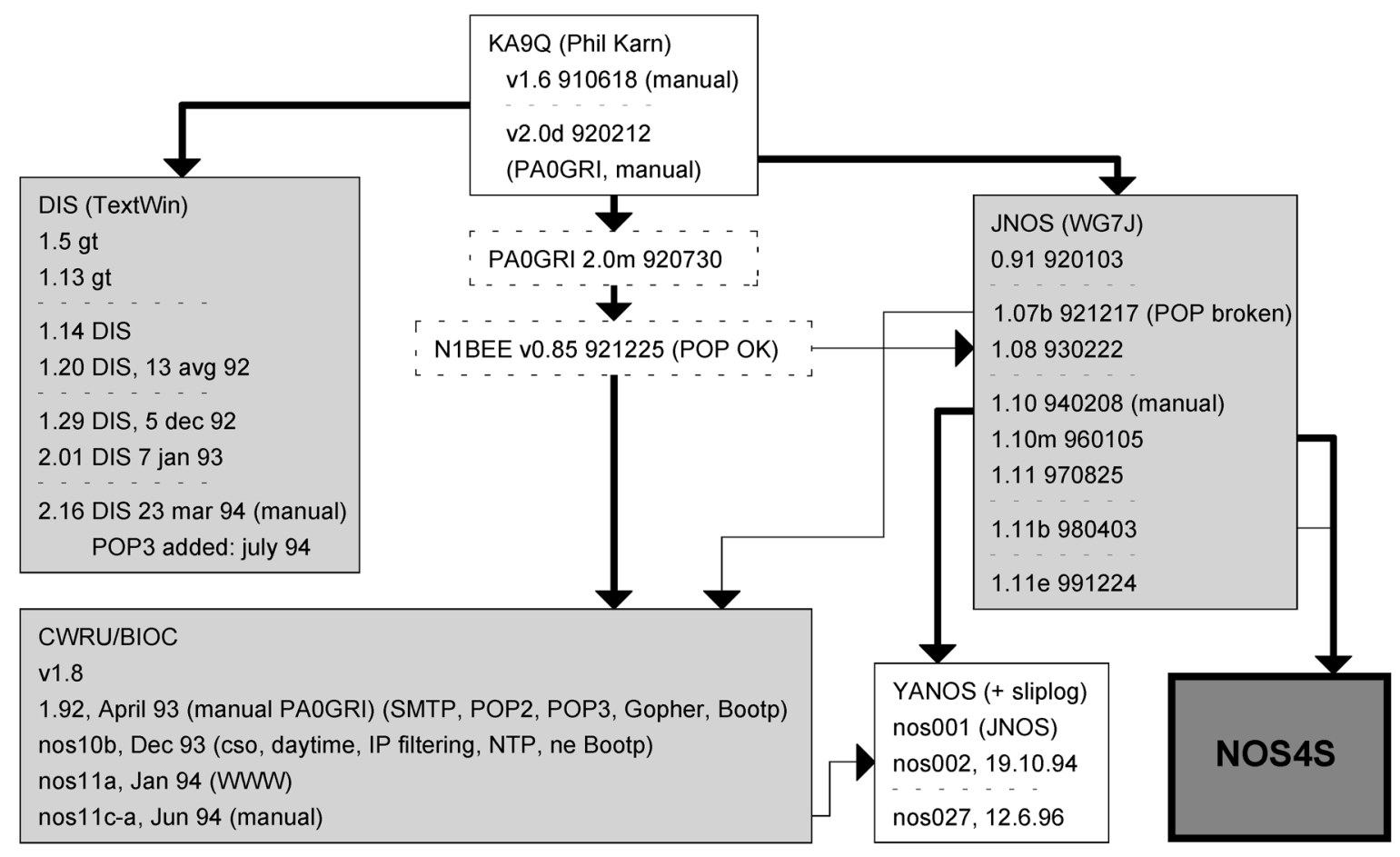

Fig. 5. KA9Q family tree. 
For NOS4S, besides a number of minor changes and corrections, the following major improvements have been made:

- On-demand PPP connection establishment. The connection is established automatically when Internet traffic is initiated from the local network to the global Internet and is closed when there is no traffic for a certain period of time. To support this functionality, the JNOS's built-in script language and command set had to be extended.

- On-demand e-mail transfer from an ESMTP server. The original JNOS' SMTP support did not include this functionality and had to be extended to use the ETRN command to request mail transfer from an ESMTP server. To use this feature, an ISP must employ an extended SMTP server (ESMTP) supporting the ETRN command (e.g. a newer version of publicly available "sendmail" for UNIX).

- Since NOS4S is (like other KA9Q family programs) relatively complex to configure properly, an auxiliary procedure has been developed to simplify this task.

Like the original JNOS, NOS4S works as a classical router and does not support proxy service or NAT. For this reason it cannot be used with a standard single-user account but needs a special LAN account with a statically assigned IP address subclass (usually a C-class or a part of it).

Besides the aforementioned, NOS4S comprises some other useful functions. The most important ones are the FTP and WWW servers, which can be employed to teach their use (e.g. preparation and publishing of WWW pages) in a classroom without a live global Internet connection.

NOS4S supports traffic filtering and access control, which is crucial for school networks. Access to local or external IP addresses or address ranges, in combination with port numbers and protocols (TCP/UDP/ICMP), can be permitted or denied. NOS4S can run on almost any kind of IBM-PC compatible computer (386 or higher) that must be equipped with a network (Ethernet) adapter and a suitable (PSTN or ISDN) dial-up modem/adapter.

\section{NOS4S in Brief:}

NOS4S offers the following functions:

- TCP/IP routing (PSTN/ISDN, on-demand dialling).

- Mail server (SMTP/POP3, on demand SMTP using ESMTP command ETRN).

- FTP and WWW server (mostly for local use).

- DNS server (local use).

- Traffic filtering.

NOS4S hardware requirements:

- IBM-PC compatible, (386 or higher).

- Hard disk (2MB for NOS4S, enough space for user files: e-mail, downloaded files, local WWW pages).

- Main memory $1 \mathrm{MB}$.

- Ethernet adapter.

- Analog modem or ISDN adapter.

The advantages of NOS4S over other solutions (e.g. MS Windows or UNIX software):

- Low hardware requirements.

- Software is free.

- Simple to install and use.

NOS4S is based on JNOS, with the following main enhancements:

- On-demand dial function together with enhanced dial-up script language.

- On-demand SMTP mail retrieval using ESMTP command ETRN.

- A number of minor modifications (including different compilation settings).

- Simplified installation procedure and setting (using a custom-written program).

An ISP (Internet Service Provider) must fulfil the following requirements:

- Provide a special LAN account with a statically assigned IP address subclass (no proxy service or NAT routing is available in NOS4S).

- Run an ESMTP e-mail server supporting the ETRN command. 


\section{Conclusions}

A program, NOS4S, has been developed for use in schools. It enables a school to connect its computer classroom to Internet using PSTN (analog) or ISDN dial-up lines. It offers an on-demand SMTP e-mail server and a POP3 email server, as well as a simple FTP and WWW server that can be used to teach these services without a live Internet connection.

NOS4S is publicly available and free. It can run under DOS on almost any kind of IBM-PC type of computer ( 386 or higher) equipped with a LAN adapter and a PSTN modem or an ISDN adapter, which makes the whole solution very affordable. The fact that NOS4S is a complete and free solution for school Internet connectivity, and can run on a low-performance IBM-PC compatible computer, is its main advantage over other existing commercial or shareware solutions. Also not pre-configured this way, NOS4S can also be used with a cable modem or ADSL connection instead of a dial-up connection. This would require installation of a second Ethernet adapter in the computer.

NOS4S has been tested in a few Slovenian schools. It has been well received and has proved to be a successful solution for Internet connection. However, another problem for wider acceptance of NOS4S has shown up: many teachers of computer-related subjects in primary schools are not familiar with the basics of network technology and their further education in this field is necessary.

NOS4S is free for non-commercial use and is available for download, both the executable and sources, from http://media.ijs.si/ igor/crii/.

\section{References}

[1] INCO-Copernicus subproject: Affordable dial-up PPP/SLIP LAN-to-Internet connection, http://media.ijs.si/ igor/crii/

[2] INCO-Copernicus project: Cooperative Research in Information Infrastructure (CRII), http://lpo.fri.uni-lj.si/crii/, http://www. newcastle.research.ec.org/ esp-syn/text/cp96-154.html

[3] RFC standards, http: //www.rfc-editor.org/, http: //www. faqs.org/

[4] D. E. COMER, Internetworking with TCP/IP, Vol I: Principles, Protocols and Architecture, Prentice Hall, Englewood Cliffs, New Jersey, 1995.

[5] Firewall Q\&A (Vicomsoft), http: //www. vicomsoft.com/knowledge/ reference/firewalls1.html

[6] SOCKS (Permeo Technologies), http://www. socks.nec.com/

[7] Network Address Translation FAQ (Vicomsoft), http: //www. vicomsoft.com/knowledge/ reference/nat.html

[8] IETF NAT Working Group, http: //www. ietf .org/html . charters/ nat-charter.html

[9] PCROUTE (Nov 1993), ftp://ftp.fu-berlin.de/pc/msdos/ network/pcroute/; INAR (V1.01, Oct 1995), ftp://ftp.fu-berlin.de/pc/msdos/ network/inar/; JNOS (V1.11f, Nov 2000), ftp://pc.louisiana.edu/pub/ham/jnos111f/; IPROUTE (V1.18, Jun 1998). http://www.mischler.com/iproute/

Received: September, 2000 Revised: November, 2001 Accepted: May, 2002

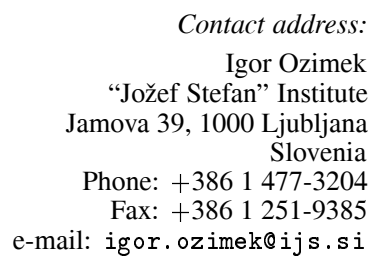

IGOR OZIMEK received his $\mathrm{BS}, \mathrm{MS}$, and $\mathrm{PhD}$ degrees in electrical en gineering from the University of Ljubljana, Slovenia in 1980, 1988, and 1993, respectively. Since 1980 he has been with "Jǒ̌ef Stefan" Institute, Ljubljana, where he works as a researcher. His current interests include digital communications, DSP processing and computer networks. 